\title{
Road Traffic Safety Concept Culture Construction Evaluation Methods Based on Fuzzy Analytical Method
}

\author{
Zhongqing Xie \\ Wuhan University of Technology, Wuhan, Hubei, China \\ xiezqwut@163.com
}

Keywords: The fuzzy analysis method; Concept of traffic safety culture; Risk prediction

Abstract: A road traffic safety culture construction concept was proposed which consisted of criterion layer and index layer. The frequency of issues that folks' focused on were analyzed based on the fuzzy analysis method, furthermore, the research results were classified by discrepancy quantification. At last, the identical degree, discrepancy degree and contrary degree of the three indexes, as well as the total discrepancy, were calculated to analyze the road traffic safety concept; and suggestions were proposed based on the classification of the evaluation index.

\section{Introduction and evaluation index}

The index system establishment is the core problem of evaluating road traffic safety concept culture construction level $^{[1]}$, which affect the credibility of evaluation results directly. Evaluation of road traffic safety concept culture construction level is a complex multi-level issue that needs to reflect the traffic safety level ${ }^{[2]}$. Domestic and foreign scholars have certain attention on road traffic safety culture construction, not enough on the concept cultural construction of road traffic safety ${ }^{[3,4]}$. There is no quantitative evaluation methods.

It is a complicated work to set up a network to evaluate the road traffic safety culture construction level ${ }^{[5,6]}$. According to the relevant state safety rules, standards and regulations, considering the road traffic safety culture construction questionnaire results, several parameters were chosen to be studied and to be utilized as evaluation indexes for the evaluation network.

Beginning with the investigation of current reality of the domestic road traffic safety culture construction; following with determining of the subject and object of the culture construction; then choosing the parameters to act as evaluation indexes; at last proposing a road traffic safety concept, which includes criterion parts and index parts, to evaluate the culture construction level objectively. This method and research results maybe a significate reference to the road traffic safety culture construction level evaluation and road traffic safety management politics.

Combining with the above on road traffic safety culture connotation and the analysis of the factors influencing the construction process, using the index system of hierarchical decomposition method, this paper sets up the concept of road traffic safety culture construction level evaluation index system as shown in table 1 .

Zhongqing Xie(1975 ), PhD candidate, xiezqwut@163.com, mainly researches college students' ideological education, clean and honest culture and traffic safety culture. 
Table 1Road traffic safety culture construction level evaluation index system

\begin{tabular}{cc}
\hline Evaluation target & Indicator system \\
\hline \multirow{3}{*}{$\begin{array}{c}\text { Tulture of traffic safety } \\
\text { concept } \\
(\mathrm{Al})\end{array}$} & benefit $\left(a_{11}\right)$ \\
\cline { 2 - 2 } & Traffic safety consciousness cognition \\
& Traffic safety risk forecast and control level $\left(a_{12}\right)$ \\
\hline
\end{tabular}

\section{Fundamental of algorithmic}

The Fuzzy mathematics analysis method was utilized in this paper, and the focusing parameters were analyzed in frequency through the SPSS software. The data specimens were classified, calculated and analyzed based on the theory of set pair analysis, the details were shown in Fig.1.

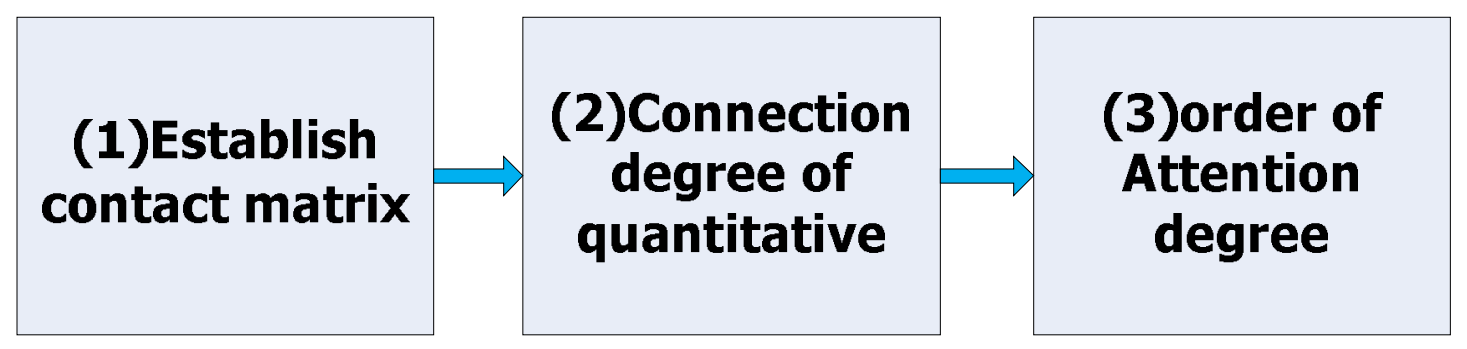

Fig.1 Procedure of calculation

\section{Identical-Discrepancy-Contrary (IDC) Matrix}

Supposed set $A$ consists of $n$ parameters, while set $B$ consists of $m$ persons' focusing issues, set $A$ and $B$ compose a pair $(A, B)[123]$ 。

It is supposed that the number of the people of identical, discrepancy and contrary of the $\mathrm{k}^{\text {th }}$ index are $S_{k}, F_{k}, P_{k}$ respectively , and the connection degree of this index can be calculated as eq. (1):

$$
\mu_{k}=a_{k}+b_{k} i+c_{k} j
$$

Where:

$$
\begin{gathered}
a_{k}=\frac{S_{k}}{m}, k=1,2, \mathrm{~L}, n \\
b_{k}=\frac{F_{k}}{m}, k=1,2, \mathrm{~L}, n \\
c_{k}=\frac{P_{k}}{m}, k=1,2, \mathrm{~L}, n
\end{gathered}
$$

So the IDC connection degree matrix which consists $n$ indexes can be written as eq. (5): 


$$
T=\left[\begin{array}{lll}
a_{1} & b_{1} & c_{1} \\
a_{2} & b_{2} & c_{2} \\
\mathrm{M} & \mathrm{M} & \mathrm{M} \\
a_{n} & b_{n} & c_{n}
\end{array}\right]
$$

\section{Quantification of discrepancy's connection degree}

The quantification of discrepancy's connection degree is the key to evaluate the focus degree. In this paper, in order to propose a optimize matrix of discrepancy's connection degree, the focus degree was quantified manually.

(1)The supreme ideal value of focus connection degree

In the IDC matrix, if $a_{0}, b_{0}$ and $c_{0}$ has the relationship as eq. (6):

$$
\left\{\begin{array}{c}
a_{0}=\max \left\{a_{k}, k=1,2, \mathrm{~L}, n\right\} \\
c_{0}=\min \left\{c_{k}=1,2, \mathrm{~L}, n\right\} \\
b_{0}=1-a_{0}-c_{0}
\end{array}\right.
$$

Therefore the supreme ideal DIC connection degree can be calculated with eq. (7):

$$
\mu_{0}=a_{0}+b_{0} i+c_{0} j
$$

(2) the optimize connection degree of focus level

Because of the $\mathrm{k}^{\text {th }}$ connection degree value $\mu_{k}=a_{k}+b_{k} i+c_{k} j$, supposed that $\mu_{k_{0}}=\mu_{0}-\mu_{k}$, it is can be calculated via DIC subtraction algorithm:

$$
\begin{aligned}
& \mu_{k 0}=\left(a_{0}-a_{k}\right)+\left(b_{0}+b_{k}\right) i- \\
& \left(c_{0}-c_{k}\right) j \underline{\underline{\Delta}} a_{k 0}+b_{k 0} i+c_{k 0} j
\end{aligned}
$$

Where: $a_{k 0}=a_{0}-a_{k}>0, c_{k 0}=c_{0}-c_{k}<0$;

Therefore $\mu_{k 0},(k=1,2, \mathrm{~L} n)$ is called the discrepancy's connection degree of the focus degree and the supreme ideal focus degree of the $\mathrm{k}^{\text {th }}$ index.

(3) The classification of the focus degree

According to theory of set pair analysis, if the value of the discrepancy's connection degree is small enough, the focus degree of that index would be high. From the definition of discrepancy's connection degree in step (2), $a_{k 0}, b_{k 0} i$ and $c_{k 0} j$ are represent the $\mathrm{k}^{\text {th }}$ index's discrepancies of identical, discrepancy and contrary respectively; in additionally, considering the conformity tests results, the $i, j$ can be supposed as eq. (7): 


$$
\begin{gathered}
i=a_{k} \times \frac{6}{5} \times 0.37, j=-1 \\
l_{k}=a_{k 0}+b_{k 0} i+c_{k 0} j=\left(a_{0}-a_{k}\right)+a_{k} \times \frac{6}{5} \times 0.37 \times\left(b_{0}+b_{k}\right)-\left(c_{0}-c_{k}\right)
\end{gathered}
$$

Where:

$l_{k}$ is the total discrepancy of focus degree and supreme ideal focus degree of the $\mathrm{k}^{\text {th }}$ index; the ranking of the focus index is based on the value of $l_{k}$.

\section{Result and analysis}

Ignoring the accumulative contribution rate more than $80 \%$ and considering the general principle, we get the sort of every index.

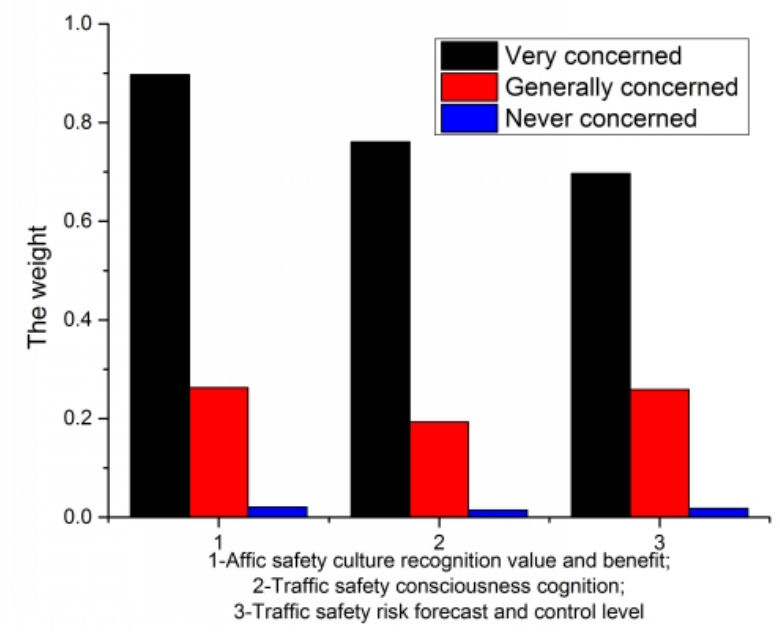

Fig. 2 The distribution of different indexes

In Fig. 2 these volumes are represent the distribution of different indexes without considering focus degree. It is indicated that all the three indexes listed in the margin of Fig.2 have the same tendency, the weight of very concerned much more than that of generally concerned which followed by never concerned.

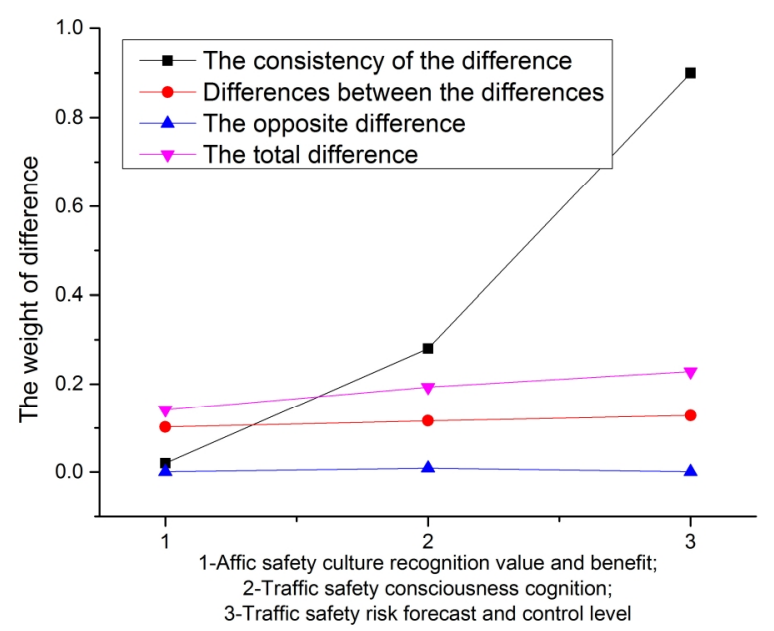

Fig.3 The distribution of different indexes' discrepancy

The weight curves of identical, discrepancy, contrary and total discrepancy are shown in Fig.3: (1) The first index-traffic safety culture recognition value and benefit composes the smallest 
proportion; the second index-traffic safety consciousness cognition composes the medium proportion; the third index-traffic safety risk forecast and control level composed the largest proportion. (2) the identical discrepancy performs a distinguish differences, while other three perform mush similar tendency with smaller discrepancy.

\section{Conclusions}

Questionnaire statistical results show that the age structure of the questionnaire survey, and the degree of culture, and road traffic safety to participate in the object conforms to the actual situation of road traffic safety culture construction in our country, which has a wide range of representative and pertinence. Combined with the questionnaire of preliminary, intuitive statistics, the main elements of road traffic safety culture construction were given. In this paper, the road traffic safety culture construction in our country's present situation and existing problems are summarized and research. Finally conclusions are as follows.

(1) Transportation concept culture construction level index rankings are: traffic safety culture value and benefit recognition is the first class, traffic safety consciousness cognition is the second class and traffic safety risk forecast and control is the third class.

(2) People's concept of road traffic safety culture is relatively weak. Associated with the "road traffic safety management level" factor questionnaire statistical results show that $65.42 \%$ of the people select "general", $65.42 \%$ of the people select "general", $25.37 \%$ of the people select "poor" and $8.24 \%$ of the people select "very good".

(3)The current road traffic safety management level of social recognition is not high. As the road traffic safety management departments and managers, there is work to further, to bear the road traffic safety as responsibility.

\section{References}

[1]X. Yang, C. Song. China's urban road traffic real-time adaptive control and management system research [J]. Journal of transportation engineering, 2001 (2) : 74-77.

[2]Z. Shao. Evaluation index system of road traffic safety culture construction level research [J].

Road traffic and safety, 2006 (6) : 17-20.

[3]Z. Shao. Construction of road traffic safety culture connotation, function and way [J]. Journal of Hubei institute for police officers, 2006 (3) : 51 and 55.

[4]Sun. Thinking about the construction of road traffic safety culture [J]. Science and technology innovation herald, 2008 (12) : 175-175.

[5]L. Chang, R. Liu. Urban traffic safety culture construction of a preliminary study [J]. Journal of comprehensive transportation, 2007 (4) : 49-52.

[6] B. Zhang. Urban pedestrian traffic safety planning study [J]. Highway traffic technology, application technology, 2007 (2). 